\title{
A Web-Based Co-Creation and User Engagement Method and Platform
}

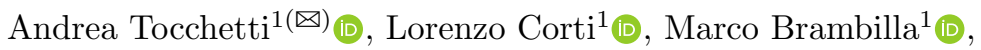 \\ and Diletta Di Marco ${ }^{2}$
}

1 Dipartimento di Elettronica, Informazione e Bioingegneria, Politecnico di Milano, Milan, Italy

\{andrea.tocchetti, lorenzo.corti, marco.brambilla\}@polimi.it

2 Dipartimento di Ingegneria Gestionale, Politecnico di Milano, Milan, Italy

diletta.marco@polimi.it

\begin{abstract}
In recent years, new methods to engage citizens in deliberative processes of governments and institutions have been studied. Such methodologies have become a necessity to assure the efficacy and longevity of policies. Several tools and solutions have been proposed while trying to achieve such a goal. The dual problem to citizen engagement is how to provide policy-makers with useful and actionable insights stemming from those processes. In this paper, we propose a research featuring a method and implementation of a crowdsourcing and co-creation technique that can provide value to both citizens and policy-makers engaged in the policy-making process. Thanks to our methodology, policy-makers can design challenges for citizens to partake, cooperate and provide their input. We also propose a web-based tool that allow citizens to participate and produce content to support the policy-making processes through a gamified interface that focuses on emotional and vision-oriented content.
\end{abstract}

Keywords: Crowdsourcing · Gamification · Co-creation • Policy-making

\section{Introduction}

Over the past decades, a new form of governance has emerged to replace adversarial and managerial modes of policy-making. Engaging citizens in decision making is gradually proving to be a new way to overcome long-lasting symptoms of a democratic deficit in modern societies, such as the reluctance to publicly state one's opinion, declining voter turnout, and the diminishing participation in public debate within institutions. Governments and institutions are struggling to understand the real impact on innovation creation of such engagement processes, how they can be adequately developed and adopted [6,11]. The theory and practice of public policy are increasingly concerned with placing the citizen at the center of policy-makers' considerations, both as target and active agent. This new route focuses on opening up governmental structures to the external

(C) Springer Nature Switzerland AG 2021

M. Brambilla et al. (Eds.): ICWE 2021, LNCS 12706, pp. 496-501, 2021.

https://doi.org/10.1007/978-3-030-74296-6_38 
environment and investigating the effect of the intensive use of data, information, and communications technology in the public sphere [7]. Public organizations are trying to learn how to encourage citizens to get involved in finding solutions to problems in the public sector for the sake of the common good. According to [1], the only way to meet and face these challenges is through the co-creation of new solutions with citizens.

This research proposes a method aimed at enabling large-scale citizen engagement and co-creation in support of policy-makers. The presented approach, as well as its implementation, is based on different principles and techniques, whose initial conceptualization has been reported in [10]. We report on our research plan and on the ongoing developments and continuous evolution with respect to the initial concepts.

\section{Related Work}

Most of the times, engaging citizens is a tough task, especially when it comes to the policy-making field. In recent years, many researchers and local administrations developed different methods and systems to achieve such a goal. Most of the developed solutions were digital, like platforms, social media and/or websites. "Love Your City" [9] allowed citizens to directly address to fellow citizens or authorities ("Addressing"), create solutions to a proposed problem ("Cocreating") and organize events ("Organizational"). "Decide" [3] was an online platform through which citizens could propose and vote new laws and opinions about the city proceedings, debate and rate how to redistribute the city's budget among projects.

Developed in the U.S.A., "MindMixer" [4] is an online platform through which citizens can express, support and comment public proposals. Its main functionalities involve submitting ideas, feedback, and photos, answering to questions on common themes and proposing their own solution to real life challenges.

Even though digital tools are more accessible and widespread, few European administrations opted for more tangible alternatives. Helsinki, Finland promoted public participation using a board game through which small teams of managers and front-line staff could learn together how to involve citizens in their work [2].

In Ovar, Portugal a method called "Participatory Budget" allowed citizens to express their support for different budget proposals [5]. The most interesting outcome was that people were asking friends and families to express their votes too. Inspired by this initiative, other cities pursued the same objective.

\section{Co-Creation and User Engagement Method}

\subsection{The Evolution of the Policy-Making Process}

In the traditional approach to policy-making citizens are perceived as passive actors. As can be evinced from Fig. 1, the active participation of citizens in the iterative formulation of a policy is able to enhance the overall outcome of such 
process by collecting direct feedback on the perceived impact of such procedure. However, this solution presents its own challenges when it comes to bridging the gap between policy-makers and citizens.

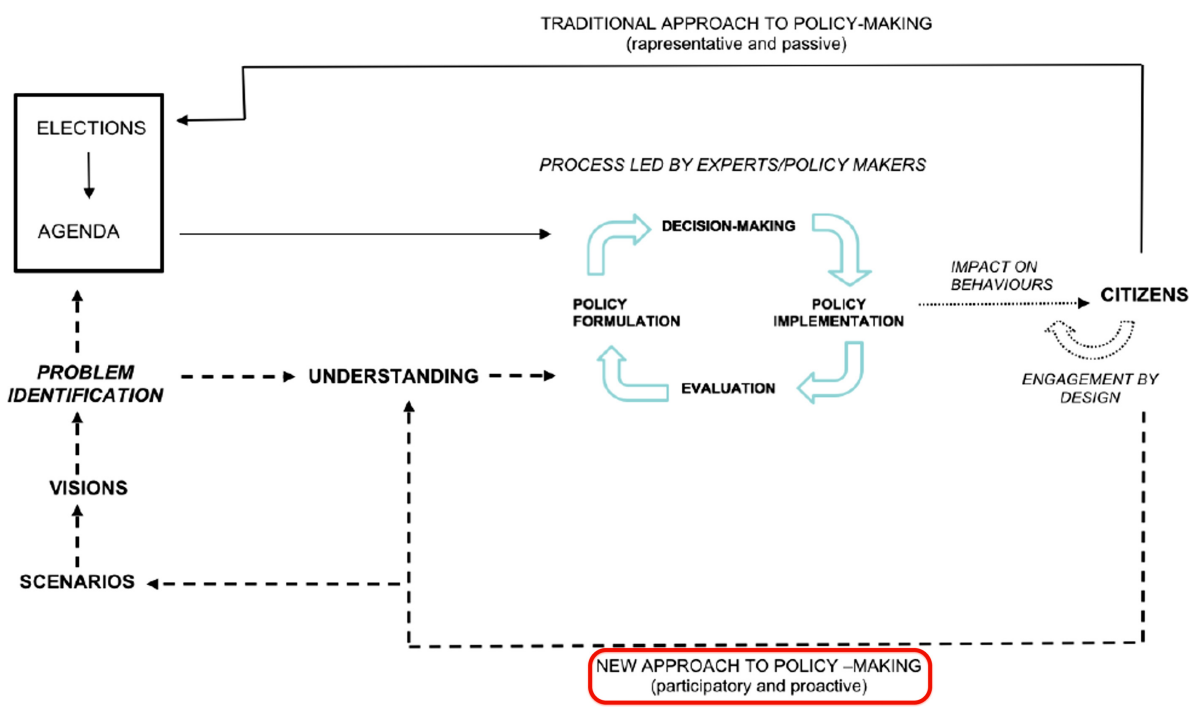

Fig. 1. Proposed co-creation process engaging both policy-makers and citizens.

\subsection{The Research Method}

In the attempt to face this problem, we devised a research plan implementing an incremental approach, where the work has been organized in four different steps:

- Definition of the theoretical model based on literature review and experts interviews;

- Realization of a paper-based implementation based on the theoretical model. The resulting physical mock-up has been tested by hosting workshops and gamified sessions, engaging experts in the social and policy-making fields to validate the engagement mechanisms. Tocchetti et al. [10] explains in details how such physical prototype was structured and how the experiment was carried out;

- Development of a digital mock-up featuring the core aspects deemed valuable based on the input of the physical phase;

- Engagement of communities of policy-making experts in the validation process of the digital mock-up. 

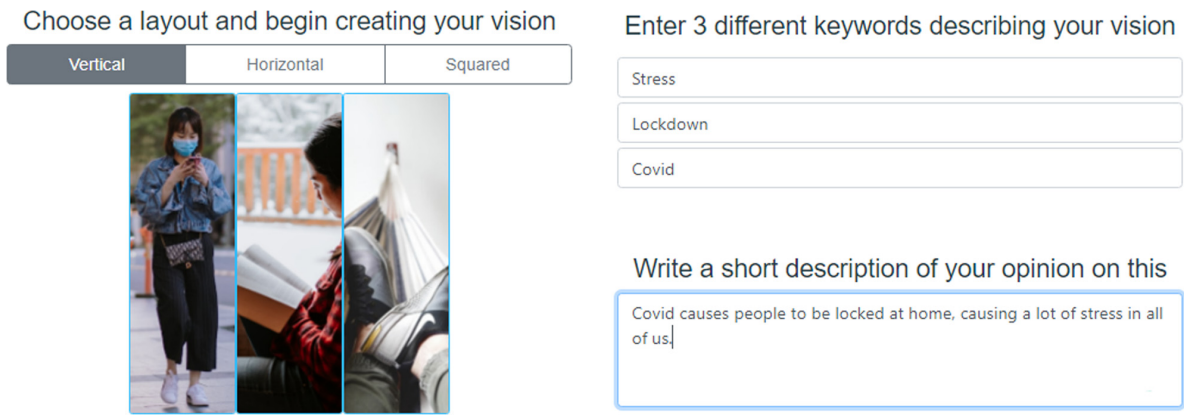

Write a short description of your opinion on this

Covid causes people to be locked at home, causing a lot of stress in all of us.

Fig. 2. Example of visual and textual content made by citizens

The preliminary feedback cycle contributed to identify some threats to the validity of our methodology. Therefore, policy-making experts were engaged in further discussion rounds. One of the aspects that has been under meticulous scrutiny is the emotional one. Due to its relevance and eventual impact on the design of the proposed interaction flow, this feature has undergone intense design cycles which led to a partial re-design of the mock-up to improve the emotional engagement of the citizens. The emotional facet has been modeled referencing the categorization of human emotions proposed by Plutchik [8]. In particular, the organization of people's emotions under eight categories, with three different intensities each, has been considered.

As most of the designed activities were successfully validated by the engaged policy-makers, a digital mock-up with the objective of improving the testing capabilities of the process has been developed. Further feedback on the final prototype have been collected to ensure that the principles validated in the physical prototype were correctly transposed into the digital one.

\subsection{The Co-creation and User Engagement Solution}

The approach and tool resulting from the research process engage citizens in a set of structured activities through which they are able to organize their thoughts in different formats. The interaction flow is structured to enable citizens to develop and convey their ideas through textual and graphical elements. In particular, the digital platform enables citizens to discuss about a variety of topics, through a series of gamified co-creation activities. The main goal is to detect moods, perceptions, and changes in the feelings of the users as they play and interact within the platform. The approach also leverages on empathy between players. Therefore, the proposed activities are aimed at structuring the thoughts of the citizens in an organized way, making them share, discuss, explore and converge on new lines of thought and visions for the future.

Thanks to its innovative content and interaction design, the proposed method is capable of capturing interesting signals from citizens about the topics of interest (Fig. 2). 


\section{Limitations and Future Work}

Even though an internal testing phase has already been carried out, it's still necessary to evaluate the effectiveness of the proposed methods in a real environment. Such assessments will engage stakeholders, policy-makers and different categories of citizens (e.g. students, citizens from a specific city, etc.). A first testing phase engaging university students will be carried out over the course of the year. Another evaluation is expected in early summer, when the citizens of an Italian city will be engaged to contribute in the decision-making process of the local administration. The proposed method will be also tested in some public events and conferences attended by policy-makers. Finally, likely the most crucial aspect, the delivery of results to policy-makers will be addressed. This final objective will be accomplished via a data visualization dashboard. Its aim is to provide a comprehensive explanation of the content shared through the platform to the policy-makers, involving not only descriptive statistics but also analytical results about topics, questionnaires, keywords and textual comments shared by citizens within the process proposed above. The data collected will also be analyzed through machine learning algorithms to extract further knowledge that can be provided to policy-makers (e.g. by classifying the citizens depending on their feelings and shared content, it would be possible to determine the polarization of the citizens with respect to a specific topic).

The final objective will be to use the approach for creating and evolving policies, around which the community will converge and gather consensus.

\section{Conclusions}

In this paper, we described one of the aspects that both local and international administrations are currently trying to deal with, namely the engagement of the citizens in co-creating solutions to current problems. As a solution to such a challenge, we briefly exhibited a methodology through which provide policymakers with insights on the thoughts of citizens, improving their decision-making capabilities. Over the rest of the year, the research will be enhanced with additional features. Furthermore, extensive experimentation will be implemented to test and validate the approach on real-world scenarios, engaging citizens and communities from different countries and with different socio-demographic characterization.

Acknowledgements. This research is partially supported by the European Commission under the H2020 framework, within project 822735 TRIGGER (TRends in Global Governance and Europe's Role) and project 101016233 PERISCOPE (Pan-European Response to the Impacts of COVID-19 and future Pandemics and Epidemics). 


\section{References}

1. Bason, C.: Leading Public Sector Innovation (Second Edition): Co-creating for a Better Society. Bristol University Press (2018). https://doi.org/10.2307/j. ctv1fxh1w

2. BloombergCities. How Helsinki uses a board game to promote public partic- ipation, January 2018. https://medium.com/@BloombergCities/how-helsinki- uses-aboard-game-to-promote-public-participation-39d580380280

3. Sam DeJohn GovLab. Beyond Protest: Examining the Decide Madrid Platform for Public Engagement. May 2018. https://blog.p2pfoundation.net/beyond-protestexamining-the-decide-madrid-platform-for-public-engagement/2018/05/09

4. Ha, A.: MindMixer Raises $\$ 17 \mathrm{M}$ To Help Governments Connect With Their Communities - TechCrunch, September 2014. https://techcrunch.com/2014/09/ 02/mindmixer-raises-17m-to-help-governments-connect-with-their-communities/

5. Mak, H.W.: A Political Success Story: Gamification as Civic Engagement Tool, January 2016. http://www.gamification.co/2016/01/11/political-successstory-gamification-civic-engagement-tool/

6. Mazzucato, M.: Mission-oriented innovation policies: challenges and opportunities. In: Industrial and Corporate Change 27.5, October 2018, pp. 803-815. ISSN: 09606491. https://doi.org/10.1093/icc/dty034

7. Misuraca, G., Pasi, G.: Landscaping digital social innovation in the EU: structuring the evidence and nurturing the science and policy debate towards a renewed agenda for social change. In: Govern- ment Information Quarterly 36.3, pp. 592-600 (2019). ISSN: 0740-624X. https://doi.org/10.1016/j.giq.2019.02.004

8. Plutchik, R.: A general psychoevolutionary theory of emotion. In: Plutchik, R., Kellerman, H. (eds.) Theories of Emotion. Academic Press, pp. 3-33 (1980). ISBN: 978-0-12-558701-3. https://doi.org/10.1016/B978-0-12-558701-3.50007-7

9. Stembert, N., Mulder, I.J.: Love your city! An interactive platform empowering citizens to turn the public domain into a participatory domain. In: International Conference Using ICT, Social Media and Mobile Technologies to Foster SelfOrganisation in Urban and Neighbourhood Governance, Delft, The Netherlands, 16-17 May 2013, May 2013

10. Tocchetti, A., Brambilla, M.: A gamified crowdsourcing framework for data-driven co-creation of policy making and social foresight. In: NeurIPS 2020 Crowd Science Workshop - CEUR Proceedings, vol. 2736, pp. 34-44 (2020)

11. Wegrich, K.: The blind spots of collaborative innovation. In: Public Management Review 21.1 (2019), pp. 12-20 (2018). 1433311. https://doi.org/10.1080/14719037 